Check for updates

Cite this: RSC Adv., 2017, 7, 31158

Received 24th April 2017

Accepted 5th June 2017

DOI: $10.1039 / \mathrm{c} 7 \mathrm{ra0} 4586 \mathrm{~g}$

rsc.li/rsc-advances

\section{Effect of organic cathode interfacial layers on efficiency and stability improvement of polymer solar cells $\dagger$}

\author{
Mingguang Li,ł Wen Zhang, \$ Honglei Wang, Lingfeng Chen, Chao Zheng \\ and Runfeng Chen (D) *
}

The cathode interfacial layer (CIL) plays a vital role in enhancing the efficiency and lifetime performance of bulk heterojunction (BHJ) polymer solar cells (PSCs). Here, we compared the use of various organic semiconducting molecules, tris(8-hydroxyquinolinato) aluminum ( $\mathrm{Alq}_{3}$ ), bathocuproine (BCP) and 1,3,5tri(m-pyrid-3-yl-phenyl)benzene (TMPyPB), as CILs in PSCs and analyzed their influence on device performance. Compared to the inorganic LiF ClL-based PSCs, significantly higher photovoltaic performance was observed by using these organic CILs in both PTB7:PC ${ }_{71} B M$ and P3HT:PC ${ }_{61} B M$ PSCs. Specifically, TmPyPB CIL-based devices exhibit superior device stability and high power conversion efficiency (PCE) up to $7.96 \%$. A systematic study on the effects of Frontier orbital energy levels, surface morphology, and electron mobility of CILs suggests that a relatively coarse interface morphology would be helpful in the formation of high-density interfacial defect states for efficient electron extraction and a high mobility is of central importance in facilitating electron collection for high PCEs. Moreover, a synergistic effect between the inorganic LiF and organic molecules in the dual-CIL could contribute to the further enhancement of PSC efficiency (8.01\%) and ambient stability. This work reveals fundamental principles in regulating the functions of CILs and would hopefully promote the investigation and development of ideal organic CILs for high-performance PSCs.

\section{Introduction}

With a remarkably high power conversion efficiency (PCE) of up to $13 \%,{ }^{1}$ polymer solar cells (PSCs) have attracted tremendous attention in both academia and industry due to their great potential and apparent advantages in fabricating renewable, low cost, lightweight, flexible, and large-area devices through low/room temperature processing and largescale production..$^{2-6}$ Besides the continuous efforts in developing high-performance photovoltaic polymers for bulk heterojunction (BHJ) solar cells in the active layer, investigations on interfacial layers between the electrode and active layer to facilitate the electron and hole collection are also essential in achieving high PCEs. The interfacial materials, either organic or inorganic, generally should have good energy-level matching between the active layer and electrodes, high conductivity,

Key Laboratory for Organic Electronics and Information Displays, Institute of Advanced Materials, Jiangsu National Synergetic Innovation Center for Advanced Materials, Nanjing University of Posts and Telecommunications, Wenyuan Road, Nanjing, 210023, P. R. China.E-mail: iamrfchen@njupt.edu.cn

$\dagger$ Electronic supplementary information (ESI) available: UV-vis absorption spectra of PTB7:PC ${ }_{71} \mathrm{BM}$ blend films; fabrication and measurement of electron-only devices; The $J-V$ curves and ambient stability characteristics of P3HT: $\mathrm{PC}_{61} \mathrm{BM}$ based PSCs. See DOI: 10.1039/c7ra04586g

\$ These authors contributed equally to this work. high optical transparency, and good thermal and chemical stability against moisture and oxygen for facile charge extraction, low resistive losses, efficient photo access to the active layer, and long-term device reliability.

Cathode interfacial layer (CIL), which is close to the metal cathode with low work function, influences significantly the electron extraction and transport processes, representing an effective way to improve the PSC performance. The contributions of CIL can be attributed to (1) suppressing the quenching of exciton at the metal electrode, (2) tuning the energylevel alignment at the interfaces for facile electron extraction, (3) protecting the active layer from damaging during the thermal deposition of metal cathode, (4) forming a passivating layer to resist oxygen and moisture diffusion. Many CIL materials including inorganic metal oxides (e.g., $\mathrm{ZnO}$ and $\left.\mathrm{TiO}_{x}\right),{ }^{7-10}$ polymer/small-molecules, ${ }^{11-13}$ fullerene-based materials, ${ }^{\mathbf{1 4}, 15}$ and other alternatives ${ }^{\mathbf{1 6}}$ have been reported and applied to optimize the device efficiency and stability. Among them, the electron transporting organic semiconductors that have been widely used in organic light emitting diodes (OLEDs), ${ }^{17-19}$ show particular advantages. ${ }^{20}$ These molecules with high electron mobility can be easily deposited by vacuum evaporation or solution-processing, generally demonstrating better PSC performance in comparison with the commonly used inorganic LiF CIL. ${ }^{21}$ However, in-depth understandings 
on the organic-metal interface and the internal correlations between CIL material properties, interface structure, contact state and type, and electron extraction and collection processes are still limited, which has hindered significantly the rational design and selection of ideal organic semiconducting CIL materials for the preparation of highperformance PSCs.

Here, we systematically investigated the relations between the organic CIL material properties and the PSC performance, focusing particularly on the effects of energy-level alignment, surface morphology, and electron mobility, based on a series of organic semiconducting molecules including tris(8hydroxyquinolinato) aluminum $\left(\mathrm{Alq}_{3}\right)$, 2,9-dimethyl-4,7diphenyl-1,10-phenanthroline (BCP), and 1,3,5-tri( $m$-pyrid-3-ylphenyl)benzene (TmPyPB) (Fig. 1a). Compared to LiF, these organic CIL molecules show improved device performance in both traditional blends of poly (3-hexylthiophene) (P3HT) and [6,6]-phenyl-C61-butyric acid methyl ester $\left(\mathrm{PC}_{61} \mathrm{BM}\right)$ and highperformance poly[[4,8-bis[(2-ethylhexyl)oxy] benzo $\left[1,2-b: 4,5-b^{\prime}\right]$ dithiophene-2,6-diyl][3-fluoro-2-[(2-ethylhexyl) carbonyl] thieno [3,4-b]thiophenediyl]] (PTB7) and [6,6]-phenyl-C71-butyric acid methyl ester ( $\left.\mathrm{PC}_{71} \mathrm{BM}\right)$ systems. TmPyPB-based PSCs has the highest device stability and PCE values up to $7.96 \%$, due to its high electron mobility and the relatively coarse surface morphology for high-density organic/metal interface defect states in facilitating the electron transport and extraction, respectively. The combination of inorganic LiF CIL and organic TmPyPB CIL further enhances hole-blocking ability to suppress charge carrier recombination and protects the underlying PSC active layer from damaging under the ambient environment, leading to improved PCE of $8.01 \%$ and device stability in dualCIL architecture.

(a)

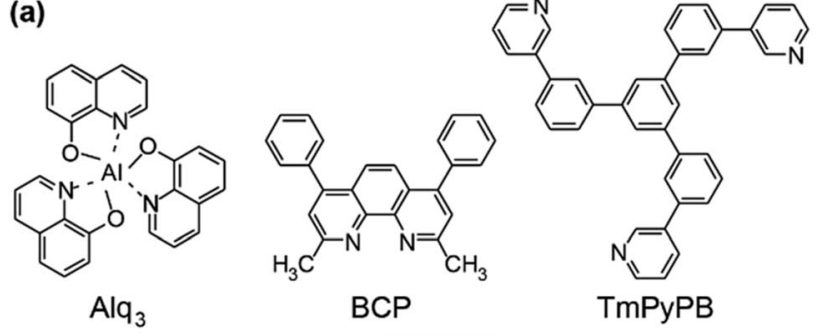

(b)

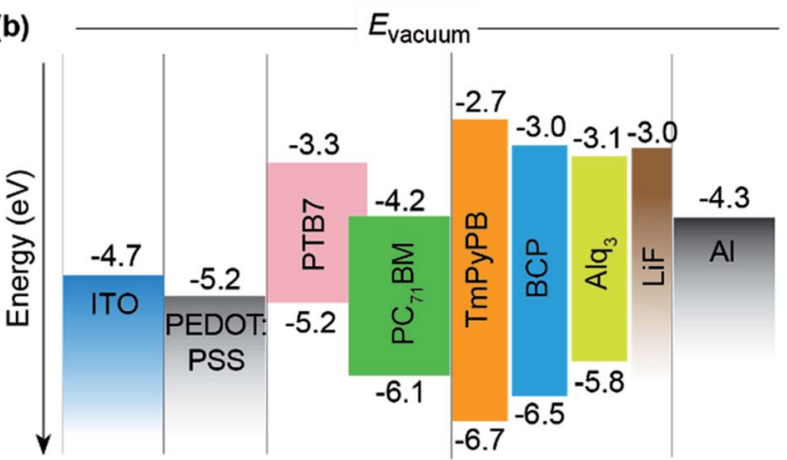

Fig. 1 (a) Molecular structures of $\mathrm{Alq}_{3}, \mathrm{BCP}$ and TmPyPB, and (b) energy levels of PSCs (in eV).

\section{Experimental}

\section{Materials}

PTB7, P3HT, $\mathrm{PC}_{71} \mathrm{BM}, \mathrm{PC}_{61} \mathrm{BM}$ and PEDOT:PSS were purchased from Luminescence Technology Corp. Chlorobenzene, o-dichlorobenzene and 1,8-diiodooctane (DIO) were from Sigma-Aldrich. Tris(8-hydroxyquinolinato) aluminum $\left(\mathrm{Alq}_{3}\right)$, bathocuproine (BCP), 1,3,5-tri(m-pyrid-3-yl-phenyl)benzene (TmPyPB) and lithium fluoride (LiF) were provided by Shanghai Han Feng Chemical Corp. All chemicals were used as obtained.

\section{Fabrication of BHJ PSCs}

The device structure was ITO/PEDOT:PSS/PTB7:PC ${ }_{71} \mathrm{BM}$ (or P3HT:PC ${ }_{61} \mathrm{BM}$ )/CIL/Al. First, the hole transporting PEDOT:PSS layer was spin-coated on top of the precleaned ITO substrate, which was treated with water, acetone and ethanol in an ultrasonic bath followed by ultraviolet (UV)-ozone treating for $25 \mathrm{~min}$ and drying at $120{ }^{\circ} \mathrm{C}$ for $30 \mathrm{~min}$. A blend of PTB7:PC ${ }_{71} \mathrm{BM}$ with the mass ratio of $1: 1.5$ in chlorobenzene solvent with $3 \mathrm{wt} \%$ DIO additives (or P3HT: $\mathrm{PC}_{61} \mathrm{BM}$ with the mass ratio of $1: 0.7$ in $o$-dichlorobenzene) was spin-coated on the surface of PEDOT:PSS layer. The thickness of the active layer was controlled to be about $100 \mathrm{~nm}$ as measured by surface profilometer. In order to investigate the influence of different CILs on the device performance, different CILs made by LiF, BCP, $\mathrm{Alq}_{3}$ and TmPyPB were deposited on the active layer by high-vacuum $\left(\sim 4 \times 10^{-4} \mathrm{~Pa}\right)$ thermal evaporation. The thickness of these CILs was optimized. Finally, Al $(\sim 100 \mathrm{~nm})$ cathode electrode was thermally deposited under a vacuum of $2 \times 10^{-4} \mathrm{~Pa}$ to complete device fabrication. These PSCs, made on ITO glass substrate, have an active area of $9 \mathrm{~mm}^{2}$.

\section{Characterization}

The morphology of the CIL layer was characterized at room temperature by atom force microscopy (AFM) using a Bruker Dimension Icon AFM equipped with Scanasyst-Air peak force tapping mode AFM tips from Bruker. UV-vis spectra were measured on a lambda 35 PerkinElmer ultraviolet-visible (UVvis) spectrophotometer. Current density-voltage $(J-V)$ characteristics of PSCs were recorded by Keithley-2400 digital source meter. Newport, Oriel Sol 3A solar simulator under simulated AM 1.5 illumination $\left(100 \mathrm{~mW} \mathrm{~cm}^{-2}\right)$ was used as solar cell characterization. DK240 (Crown. Tech. Inc) was used for external quantum efficiency (EQE) measurement.

\section{Results and discussion}

Three typical OLED electron transport materials of $\mathrm{Alq}_{3}$, BCP and TmPyPB with high transparency in visible light region and various Frontier orbital energy levels were selected to investigate the effect of organic CIL on the performance of PSCs (Fig. 1b). Being sandwiched between the organic PSC active layer and the metal cathode as a buffer, the CIL should bridge the organic and inorganic layers together with facilitated electron extraction and transport processes for enhanced electron collection and suppressed hole transportation. Therefore, according to the 
Frontier orbital energy level analysis, appropriate CIL material should have the deep the highest occupied molecular orbital (HOMO) to block the hole injection and low the lowest unoccupied molecular orbital (LUMO) to support the efficient electron extraction and transportation; also, a larger bandgap than that of the blend polymer is required to prevent the charge carrier recombination in the CIL layer.

Based on the typical BHJ PSC structure of ITO/PEDOT:PSS/ PTB7:PC ${ }_{71} \mathrm{BM} / \mathrm{CIL} / \mathrm{Al}$ (inset of Fig. 2) with an active area of
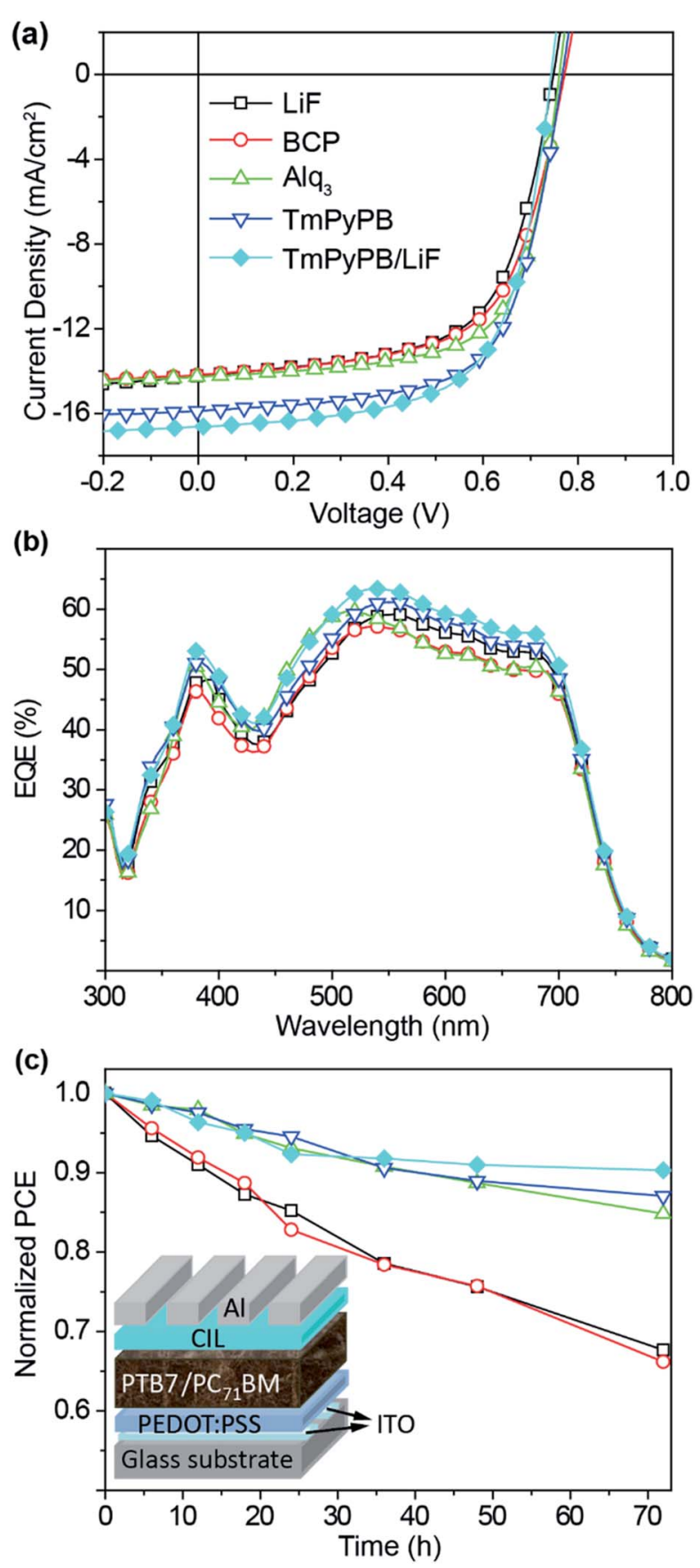

Fig. 2 (a) Current-voltage ( $J-V$ curves, (b) EQE spectra, and (c) airstability characteristics of PTB7:PC ${ }_{71}$ BM-based PSCs with different CILs under ambient environment. Inset shows the schematic representation of the PSC structure.
$9 \mathrm{~mm}^{2}$ (Fig. S1 $\dagger$ ), the influence of the organic CILs on the photovoltaic properties were studied. These devices exhibit similar open-circuit voltages $\left(V_{\text {oc }}\right)$ of $0.75 \pm 0.02 \mathrm{~V}$, but varied short-circuit currents $\left(J_{\mathrm{sc}}\right)$ from 14.18 to $16.62 \mathrm{~mA} \mathrm{~cm}^{-2}$ and PCE from $6.84 \%$ to $8.01 \%$ after the incorporation of different CILs. Compared to the LiF-only CIL reference device, the use of organic CILs results in comparable $V_{\mathrm{oc}}$ and fill factor (FF) but significantly increased $J_{\mathrm{sc}}$, and thus improved PCE in the order of $\mathrm{LiF}<\mathrm{BCP}<\mathrm{Alq}_{3}<\mathrm{TmPyPB}<\mathrm{TmPyPB} / \mathrm{LiF}$; this selectively enhanced $J_{\mathrm{sc}}$ can also be found in the literature using other organic CIL molecules (Fig. 2a). ${ }^{20,22,23}$ As shown in Table 1, series resistance $\left(R_{\mathrm{S}}\right)$ decreases from $7.5 \Omega \mathrm{cm}^{2}$ for LiF-only device to $5.3 \Omega \mathrm{cm}^{2}$ for TmPyPB/LiF dual-CIL device, indicating a reduced semiconductor bulk resistance and a better metal/ semiconductor interface connection, which should be responsible for the enhanced $J_{\mathrm{sc}}{ }^{24}$ As to the slightly varied $V_{\mathrm{oc}}$, different natures of these CIL materials may influence the charge recombination dynamics of the photovoltaic materials in the active layer, leading to disturbed $V_{\mathrm{oc}}$ in a small range of variation. ${ }^{25}$

With the deepest HOMO of TmPyPB CIL to block effectively the hole diffusion towards the cathode side, the highest $J_{\mathrm{sc}}$ and PCE values were resulted, and these values can be further improved to $16.62 \mathrm{~mA} \mathrm{~cm} \mathrm{~cm}^{-2}$ and $8.01 \%$ when using the TmPyPB/LiF dual-CIL. The low PSC performance of $\mathrm{Alq}_{3} \mathrm{CIL}$ should be related to its unmatched HOMO, which is too low (lower than that of $\mathrm{PC}_{61} \mathrm{BM}$ in the active layer) to prevent the charge recombination in CIL. The external quantum efficiency (EQE) spectra of the five PSC devices with different CILs were measured (Fig. 2b). It was found that the EQE spectrum with similar sharp enhances in the whole wavelength range in a same order to the $J_{\text {sc }}$ enhancement of $\mathrm{BCP}<\mathrm{Alq}_{3}<\mathrm{TmPyPB}<$ TmPyPB/LiF. Specifically, LiF-only device exhibits a maximum of $\sim 59 \%$ at $560 \mathrm{~nm}$, while that of TmPyPB/LiF dual-CIL device reaches a maximum of $\sim 63 \%$ at $540 \mathrm{~nm}$ with much more significant improvement at the wavelength band of 350$680 \mathrm{~nm}$. To reveal the origin of the increased $J_{\mathrm{sc}}$, EQE and PCEs using various organic CILs, the UV-vis absorption of PTB7:PC ${ }_{71} \mathrm{BM}$ blend films with CILs were characterized (Fig. S2 $\dagger$ ). Negligible absorption difference of these films was observed, suggesting there are limited effects of CILs on the light harvesting for increased $J_{\mathrm{sc}}$ and PCE.

As to the influence of CILs on the PSC stability (Fig. 2c), the LiF-only device suffered from rapid degradation in PCE value within $72 \mathrm{~h}$. In contrast, TmPyPB-only device and TmPyPB/LiF dual-CIL device exhibit significant lifetime improvement, maintaining up to $90 \%$ of its original value after $72 \mathrm{~h}$ in air without any encapsulation. Thus, air-stability modification is identified to be correlated with the introduced organic CIL, which could effectively block the oxygen and water from permeating through the CIL. The positive effect of $\mathrm{Alq}_{3}$ and TmPyPB on the device stability has also been reported in other PSC systems. ${ }^{22,26}$ However, it should be noted that the introduction of BCP layer cannot contribute much to the improvement of device stability, probably because BCP thin film can readily crystallize especially in the presence of moisture, ${ }^{22,27}$ leading to the rapid degradation of device performance. 
Table 1 Device performance of PTB7:PC ${ }_{71} B M$ and P3HT:PC ${ }_{61} B M$ based PSCs with different CILs

\begin{tabular}{|c|c|c|c|c|c|c|c|}
\hline System & CILs & $V_{\mathrm{oc}}[\mathrm{V}]$ & $J_{\mathrm{sc}}\left[\mathrm{mA} \mathrm{cm}^{-2}\right]$ & $\mathrm{FF}[\%]$ & PCE $[\%]$ & $R_{\mathrm{s}}\left[\Omega \mathrm{cm}^{2}\right]$ & $R_{\mathrm{sh}}\left[\Omega \mathrm{cm}^{2}\right]$ \\
\hline \multirow[t]{5}{*}{ PTB7:PC ${ }_{71} \mathrm{BM}$} & $\mathrm{LiF}$ & 0.74 & 14.22 & 62.8 & 6.68 & 7.5 & 506.9 \\
\hline & $\mathrm{BCP}$ & 0.77 & 14.18 & 62.6 & 6.84 & 7.9 & 664.7 \\
\hline & $\mathrm{Alq}_{3}$ & 0.76 & 14.27 & 66.9 & 7.26 & 6.1 & 879.4 \\
\hline & TmPyPB & 0.77 & 15.89 & 64.9 & 7.96 & 6.6 & 808.5 \\
\hline & TmPyPB/LiF & 0.73 & 16.62 & 66.0 & 8.01 & 5.3 & 857.9 \\
\hline \multirow[t]{5}{*}{ P3HT:PC ${ }_{61}$ BM } & $\mathrm{LiF}$ & 0.64 & 8.41 & 59.7 & 3.27 & 11.5 & 547.1 \\
\hline & $\mathrm{BCP}$ & 0.65 & 8.86 & 57.0 & 3.32 & 11.1 & 480.6 \\
\hline & $\mathrm{Alq}_{3}$ & 0.63 & 9.34 & 60.1 & 3.53 & 11.0 & 460.4 \\
\hline & TmPyPB & 0.63 & 9.74 & 58.0 & 3.60 & 11.2 & 472.2 \\
\hline & TmPyPB/LiF & 0.63 & 9.80 & 62.2 & 3.80 & 10.5 & 531.5 \\
\hline
\end{tabular}

To further elucidate the factors responsible for the enhanced $J_{\text {sc }}$ value, the AFM height images of different CILs over PTB7:PC ${ }_{71} \mathrm{BM}$ blend films were investigated (Fig. 3), because it is well-known that interface morphology may influence the organic-metal contact, charge transport, and optical property significantly ${ }^{28,29}$ After deposition of a thin layer of LiF (1 nm), the CIL exhibits very uniform surface morphology with rms roughness of $3.29 \mathrm{~nm}$ (Fig. 3a), which is very close to that of the
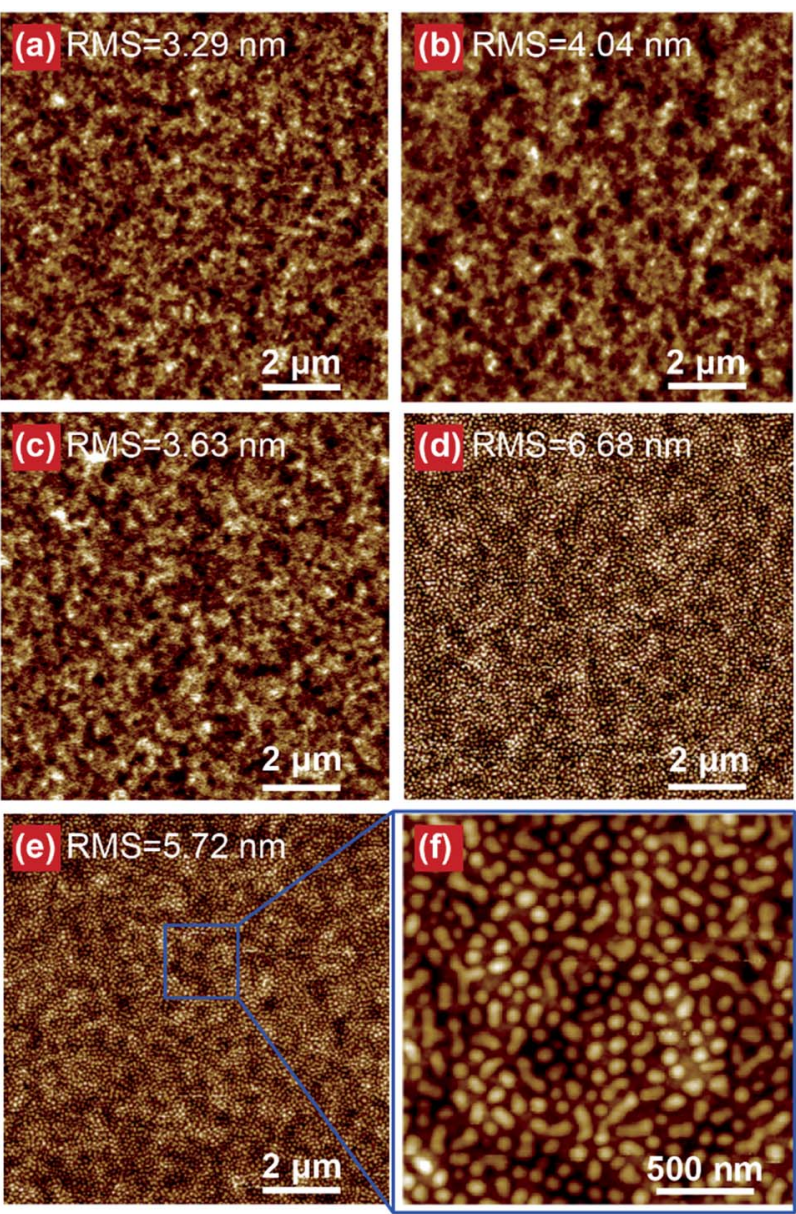

Fig. 3 AFM height images $(10.0 \mu \mathrm{m} \times 10.0 \mu \mathrm{m})$ of (a) LiF, (b) BCP, (c) Alq $_{3}$, (d) TmPyPB and (e) TmPyPB/LiF dual CILs on PTB7:PC ${ }_{71}$ BM based PSCs. (f) The magnified AFM images $(2.0 \mu \mathrm{m} \times 2.0 \mu \mathrm{m})$ of $(\mathrm{e})$. original surface of $\mathrm{PTB} 7: \mathrm{PC}_{71} \mathrm{BM}$ active layer (Fig. S3 $\dagger$ ). The vacuum deposition of organic semiconductor CILs of BCP (5 $\mathrm{nm}$ ) and $\mathrm{Alq}_{3}(5 \mathrm{~nm})$ (Fig. $3 \mathrm{~b}$ and c) also show the uniform surface morphology with only slightly increased film roughness (rms) of 4.04 and $3.63 \mathrm{~nm}$, respectively. In contrast, the film morphology changes significantly when TmPyPB was deposited as a CIL $(8 \mathrm{~nm})$ on the active layer (Fig. $3 \mathrm{~d})$. As magnified in Fig. S4, $\uparrow$ "island" structure with the diameter about $100 \mathrm{~nm}$ was observed and the rms roughness reached $6.68 \mathrm{~nm}$. When another LiF CIL ( $1 \mathrm{~nm})$ was deposited onto the TmPyPB CIL to form the dual-CIL (Fig. 3e and f), the "island" structure remains with similar size but decreased film roughness $(\mathrm{rms}=5.72 \mathrm{~nm})$.

As previously reported, the photogenerated electrons can only be transported from the LUMO of acceptor molecules into the metal cathode through the defect states below the LUMO of CIL materials when the LUMO of acceptor molecules lies below that of CIL materials by $\sim 1.0 \mathrm{eV} .^{30}$ The so-called "defect states" in the CILs are induced by the thermalization of the hot Al atoms during deposition of the electrode. ${ }^{31}$ In this sense, the rough surface morphology with high density of "island" structures, which can maximize the contact area between CIL and Al cathode, are expected to be advantageous for the creation of higher density of defect states responsible for the electron extraction. ${ }^{32}$ This should be an important reason for the highest PSC performance observed when using the TmPyPB-based CIL.

Although the Frontier orbital energy level analysis of the PSC layers gives a good prediction of the highest PSC performance by using TmPyPB-based CIL, it cannot explain the poor performance using BCP CIL ( $\mathrm{PCE}=6.84 \%$ ), because $\mathrm{BCP}$ has very close HOMO $(-6.5 \mathrm{eV})$ and LUMO $(-3.0 \mathrm{eV})$ to those of TmPyPB $(-6.7 \mathrm{eV}$ and $-2.7 \mathrm{eV})$ and its interface morphology is also similar to that of $\mathrm{Alq}_{3}$. Considering central role of CIL in transporting electron and blocking hole from the active layer to the metal cathode, the charge mobility of the CIL materials must be crucial in influencing CIL performance. From the literature reports (Table S1 $\dagger$ ), the highest electron mobility can be found in TmPyPB $\left(1.0 \times 10^{-3} \mathrm{~cm}^{2} \mathrm{~V}^{-1} \mathrm{~S}^{-1}\right)$, ${ }^{22}$ which is coincident with our measurements based on electron-only devices using the space charge limited current (SCLC) model (Fig. S5 $\dagger$ ). Therefore, a relatively low electron mobility $(5.0 \times$ $10^{-6} \mathrm{~cm}^{2} \mathrm{~V}^{-1} \mathrm{~S}^{-1}$ ) of $\mathrm{BCP}^{33}$ should be a main reason for its lowest PCE, although BCP has acceptable Frontier orbital energy levels and uniform surface morphology. The high 
electron mobility of TmPyPB guarantees the efficient electron transport during the charge collection process for the high PCEs.

The $J-V$ characteristics of $\mathrm{PTB} 7: \mathrm{PC}_{71} \mathrm{BM}$ PSCs were further measured in the dark condition (Fig. 4a) to investigate the effective injection current that may be responsible for the prominently enhanced PCEs of TmPyPB-based devices. The high rectification factor (23 288 at $\pm 1.5 \mathrm{~V}$ ) and low leakage current $\left(6.62 \times 10^{-3} \mathrm{~mA} \mathrm{~cm} \mathrm{~cm}^{-2}\right.$ at $\left.-1.5 \mathrm{~V}\right)$ of the TmPyPB-only device clearly implies its excellent diode property (i.e. transporting electrons and blocking the holes, as shown in Fig. 4b). Similar $J-V$ property under dark condition was observed for TmPyPB/LiF dual-CIL device with rectification factor up to 26008 at $\pm 1.5 \mathrm{~V}$. Compared to LiF-only device with rectification factor around 355 at $\pm 1.5 \mathrm{~V}$, the introduction of $\mathrm{Alq}_{3} \mathrm{CIL}$ also results in enhanced diode property to some extent, but the BCP CIL shows almost no diode character enhancement. Since the shunt resistance $\left(R_{\mathrm{sh}}\right)$ of PSC devices usually reflects the degree of leakage current, these results observed in dark condition can be also supported by the variation of $R_{\mathrm{sh}}$ shown in Table 1 . The introduction of organic CILs results in exceptionally enlarged $R_{\text {sh }}$ from $506.9 \Omega \mathrm{cm}^{2}$ for LiF to more than $800 \Omega \mathrm{cm}^{2}(879.4 \Omega$ $\mathrm{cm}^{2}$ for $\mathrm{Alq}_{3}, 808.5 \Omega \mathrm{cm}^{2}$ for TmPyPB and $857.9 \Omega \mathrm{cm}^{2}$ for TmPyPB/LiF), demonstrating clearly the positive effect of organic CILs in restraining the leakage current under reverse bias. ${ }^{24}$ These observations, which are in line with the electron mobility study, suggest again the profound impacts of electron mobility on the performance of CILs for PSCs.
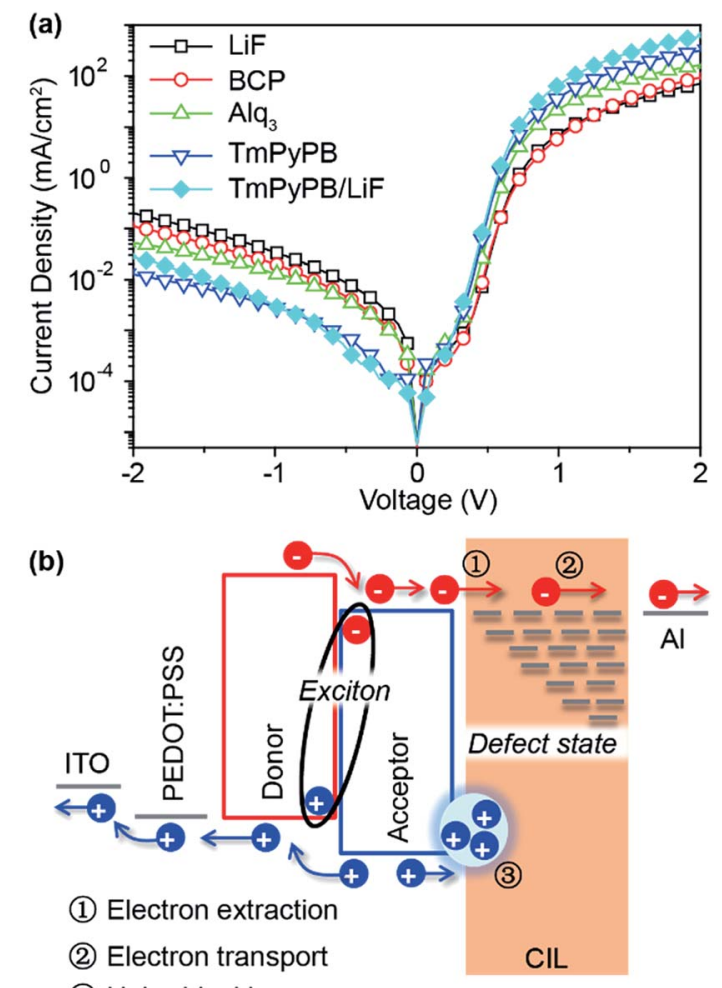

(3) Hole-blocking

Fig. 4 (a) J-V characteristics of PSCs with different CILs measured under dark conditions. (b) The schematic representation of the role of $\mathrm{CIL}$ on charge collection.
The enhancement effects of organic CILs on PSC performance were also observed in classical P3HT:PC 61 BM system. Similar rules in influencing device performance and air-stability are still valid in this different PSC system (Fig. S6†). From the summarized photovoltaic parameters in Table 1 , TmPyPB/LiF dual-CIL, which has the best device performance, can benefit from both sides of organic and inorganic parts as well as their synergistic effects, offering a high-performance organic/ inorganic CIL configuration to improve charge carrier extraction and transportation for varied PSCs with improved PCEs.

The successful attempt in using organic CILs and organic/ inorganic dual-CILs to enhance the PSC performance (Fig. 4b) makes it possible to shed light on selecting or designing suitable organic CIL materials. An ideal CIL material should have (1) sufficient defect states to facilitate the electron extraction from the acceptor material, (2) high electron mobility to transport the extracted electron to the surface of metal cathode, (3) deep HOMO and low hole mobility to block the hole injection to the CIL and transport to the cathode. To this end, a relatively rough CIL morphology is expected to produce more defect states, while the LUMO energy level has limited effects on the electron extraction; high intrinsic electron mobility of the CIL material is important for facile electron transport to the cathode; deep HOMO of the CIL material is required to block the hole injection from the active layer and to prevent the recombination in the CIL; the CIL layer should be stable and compact to protect the active layer by resisting oxygen and moisture diffusion for high device stability.

\section{Conclusions}

Three organic semiconducting materials have been tested as CILs in both traditional P3HT: $\mathrm{PC}_{61} \mathrm{BM}$ and high-performance PTB7:PC ${ }_{71} \mathrm{BM}$ PSCs. Compared to the reference LiF-based device, effectively improved efficiency and stability of the organic CIL-based devices have been observed. Especially, the TmPyPB-based devices achieve a prominent enhanced PCE value of $7.96 \%$ in single-CIL and $8.01 \%$ in dual-CIL devices with excellent stability. Based on a systematic study on the relations between the PSC performance and the organic CIL material properties including Frontier orbital energy levels, layer morphology and electron mobility, a basic guideline towards the design and applications of organic CIL materials has been proposed. It suggests that besides other requirements of CIL molecules, a relatively rough CIL morphology is favorable to produce more defect states for the enhanced electron extraction, while high intrinsic electron mobility and deep HOMO of TmPyPB material are desired to effectively transport electron and block hole from the active layer to the metal cathode, respectively. These findings may not only update the understandings of effects of CILs on the PSC efficiency and stability, but also provide comprehensive guidelines for the development of high-performance organic CIL molecules with optimal PSC applications.

\section{Acknowledgements}

This study was supported in part by the National Natural Science Foundation of China (21304049 and 21674049), Qing 
Lan project of Jiangsu province, Science Fund for Distinguished Young Scholars of Jiangsu Province of China (BK20150041), and Natural Science Foundation of Jiangsu Province of China (BK20160891).

\section{Notes and references}

1 W. R. Mateker and M. D. McGehee, Adv. Mater., 2017, 29, 1603940.

2 G. Yu, J. Gao, J. C. Hummelen, F. Wudl and A. J. Heeger, Science, 1995, 270, 1789.

3 G. Dennler, M. C. Scharber and C. J. Brabec, Adv. Mater., 2009, 21, 1323.

4 F. C. Krebs, Sol. Energy Mater. Sol. Cells, 2009, 93, 465.

5 R. F. Chen, Y. Wang, T. Chen, H. H. Li, C. H. Zheng, K. Yuan, Z. X. Wang, Y. Tao, C. Zheng and W. Huang, J. Phys. Chem. B, 2015, 119, 583.

6 R. F. Chen, C. H. Zheng, C. Li, H. H. Li, Z. X. Wang, Y. T. Tang, H. J. Jiang, Z. A. Tan and W. Huang, Polym. Chem., 2016, 7, 780.

7 S. Ben Dkhil, D. Duche, M. Gaceur, A. K. Thakur, F. B. Aboura, L. Escoubas, J.-J. Simon, A. Guerrero, J. Bisquert, G. Garcia-Belmonte, Q. Bao, M. Fahlman, C. Videlot-Ackermann, O. Margeat and J. Ackermann, Adv. Energy Mater., 2014, 4, 1400805.

8 S. H. Park, A. Roy, S. Beaupre, S. Cho, N. Coates, J. S. Moon, D. Moses, M. Leclerc, K. Lee and A. J. Heeger, Nat. Photonics, 2009, 3, 297.

9 Z. Yin, Q. Zheng, S.-C. Chen, J. Li, D. Cai, Y. Ma and J. Wei, Nano Res., 2015, 8, 456.

10 J. Huang, Z. Yin and Q. Zheng, Energy Environ. Sci., 2011, 4, 3861.

11 Z. He, C. Zhong, X. Huang, W.-Y. Wong, H. Wu, L. Chen, S. Su and Y. Cao, Adv. Mater., 2011, 23, 4636.

12 L. Nian, W. Q. Zhang, N. Zhu, L. L. Liu, Z. Q. Xie, H. B. Wu, F. Wuerthner and Y. G. Ma, J. Am. Chem. Soc., 2015, 137, 6995.

13 Z. G. Zhang, B. Y. Qi, Z. W. Jin, D. Chi, Z. Qi, Y. F. Li and J. Z. Wang, Energy Environ. Sci., 2014, 7, 1966.
14 Z. A. Page, Y. Liu, V. V. Duzhko, T. P. Russell and T. Emrick, Science, 2014, 346, 441.

15 Y. Liu, Z. Page, S. Ferdous, F. Liu, P. Kim, T. Emrick and T. Russell, Adv. Energy Mater., 2015, 5, 1500405.

16 M. Vasilopoulou, A. M. Douvas, L. C. Palilis, S. Kennou and P. Argitis, J. Am. Chem. Soc., 2015, 137, 6844.

17 Y. Tao, L. Xu, Z. Zhang, R. Chen, H. Li, H. Xu, C. Zheng and W. Huang, J. Am. Chem. Soc., 2016, 138, 9655.

18 Y. Tao, X. Guo, L. Hao, R. Chen, H. Li, Y. Chen, X. Zhang, W. Lai and W. Huang, Adv. Mater., 2015, 27, 6939.

19 Z. An, C. Zheng, Y. Tao, R. Chen, H. Shi, T. Chen, Z. Wang, H. Li, R. Deng, X. Liu and W. Huang, Nat. Mater., 2015, 14, 685.

20 L. Xiao, Z. Chen, B. Qu, J. Luo, S. Kong, Q. Gong and J. Kido, Adv. Mater., 2011, 23, 926.

21 Z. Yin, J. Wei and Q. Zheng, Adv. Sci., 2016, 3, 1500362.

22 H.-W. Lin, C.-W. Lu, L.-Y. Lin, Y.-H. Chen, W.-C. Lin, K.-T. Wong and F. Lin, J. Mater. Chem. A, 2013, 1, 1770.

23 A. Singh, A. Dey, D. Das and P. K. Iyer, ACS Appl. Mater. Interfaces, 2016, 8, 10904.

24 R. A. J. Janssen and J. Nelson, Adv. Mater., 2013, 25, 1847.

25 D. Credgington and J. R. Durrant, J. Phys. Chem. Lett., 2012, 3, 1465.

26 C. Zhang, P. Zhang, X. Xu, Y. Dang, X. Chen and B. Kang, Mater. Lett., 2016, 164, 591.

27 P. Peumans, A. Yakimov and S. R. Forrest, J. Appl. Phys., 2003, 93, 3693.

28 T.-H. Lai, S.-W. Tsang, J. R. Manders, S. Chen and F. So, Mater. Today, 2013, 16, 424.

29 S.-I. Na, S.-S. Kim, J. Jo, S.-H. Oh, J. Kim and D.-Y. Kim, Adv. Funct. Mater., 2008, 18, 3956.

30 P. Peumans and S. R. Forrest, Appl. Phys. Lett., 2001, 79, 126.

31 Y. Hirose, A. Kahn, V. Aristov, P. Soukiassian, V. Bulovic and S. R. Forrest, Phys. Rev. B: Condens. Matter Mater. Phys., 1996, 54, 13748.

32 T. Earmme and S. A. Jenekhe, J. Mater. Chem., 2012, 22, 4660.

33 J. Yu, N. Wang, Y. Zang and Y. Jiang, Sol. Energy Mater. Sol. Cells, 2011, 95, 664. 